\title{
Assessment and Comparison of Functionalities of Telemedical Applications
}

\author{
Malgorzata Plechawska-Wójcik \\ Lublin University of Technology, \\ Nadbystrzycka 36B, Lublin, 20-618, Poland
}

\author{
Joanna Rybka \\ Collegium Medicum UMK, \\ Bydgoszcz, 08-092, Poland \\ Hasselt University, Belgium, Research Center for \\ Entrepreneurship and Innovation
}

\begin{abstract}
Medical services on the Internet are modern alternative to traditional medical care supporting many functions of healthcare process. In order to improve quality and usability of upcoming tools, evaluation of existing solutions may provide valuable insight for further developments. Therefore the objective of this study was to analyze functionalities of selected telemedical applications. Our analysis provided overview of most widely- used tools and their functionalities. We discriminated three important groups of telemedical applications which were further characterized by ascribing specific functionalities considering also main and supporting role of these functionalities in each group. Our analysis resulted in extensive yet non-exhaustive list of functionalities. Based on data ontology commonly used in medical applications we distinguish three main groups of applications: Communication and social networks, Self-observation, monitoring and diagnosis, Support for medical professionals. Functional analysis for each group of medical applications was performed based on selected cases. According to the findings from our study the major criterion that allows to disentangle the heterogeneity of medical application functionalities is professionalization of the medical application. It is plausible that in future different groups of applications will become even less distinguishable due to increasing applicability of social network features in web applications but also because of increasing popularity of ehealth add-ons and tools which are becoming increasingly integrated with complementary solutions. We also identified some unexplored potential of medical applications in the field of disease prevention.
\end{abstract}

\section{General Terms}

Information Personalization, Data modeling, Data ontology

\section{Keywords}

Model medical application, ontology, functionalities, disease monitoring, telecare

\section{INTRODUCTION}

Nowadays most of the countries are facing many problems with their healthcare systems such as lack of professional personnel in medical care, low efficiency of healthcare services, low financial resources and increasing demand of healthcare from aging society. Modern alternative to traditional medical care are healthcare services on the Internet which has become less expensive, more ubiquitous and more accessible, even to people in remote areas.

The term "telemedicine" is known as "the use of audio, video and other telecommunications and electronic information processing technologies for the transmission of information and the data relevant to the diagnosis and treatment of medical conditions, to provide health services at distant sites" [1]. American Telemedicine Association defines "telemedicine" as "the use of medical information exchange from one site to another via electronic communications to improve patients' health status" [2]. Technologies used in this particular technique of communication are mostly: fax, e-mail, SMS, videoconferencing and medical device telemetry. The first generation of telemedicine solutions appeared in late 1960s and early 1970s. However these first attempts failed as technologies were seen unreliable and expensive, research in this area was underfunded and challenged with unrealistic expectations for results and limited acceptance by mainstream medicine [3]. The second generation of telemedicine tools was started in 1990s and its success is much more noticeable.

Since then, the term "telemedicine" has evolved into telehealth and afterwards - e-Health. According to S. Koch "e-healh is an emerging field in the intersection of medical informatics, public health and business, referring to health services and information delivered or enhanced through the Internet and related technologies. In a broader sense, the term characterizes not only a technical development, but also a state-of-mind, a way of thinking, an attitude, and a commitment for networked, global thinking, to improve healthcare locally, regionally and worldwide by using information and communication technology" [4]. The newest trend in telemedicine is telehomecare visit, which consists of self-management of chronic illnesses. Home e-healh includes telehomecare and smart houses, which are equipped with high-technology monitoring systems.

Another definition states that telemedicine is a modality for delivering health when two sites can communicate in many ways including client-provider, provider-provider, clientclient communication. Often they cannot meet each other because of geography, convenience, or other practical issues. Telemedicine tools not only support communication but also many functions of healthcare process, including the following [4]:

Information transfer,
Informed decision making,
Development and management of healthy
behaviours,
Sharing peer information
Providing emotional support,
Enabling self-help and self-care,
Creating demand for health services.

Growing demand for telemedicine solutions increased the availability of different tools and solutions which apply IT technology to support variety of processes alongside healthcare value chain. Amongst these tools and solutions one 
of the most dynamically increasing are mobile and internet applications.

The paper is organized as follows: second chapter gives details on methods applied in this study including functional analysis and data ontology analysis, the results are described in third chapter, followed by discussion in chapter four.

\section{TELEMEDICAL APPLICATIONS}

Medical applications are getting more and more popular among both: physicians and patients. There are several types of such applications. Some of them are highly specialized, dedicated to support diagnosis and treatments, using sophisticated data analysis methods whereas others serve more educational purposes [5]. However, interactive applications dedicated to both, doctors and patients, gain the greatest popularity nowadays [6]. There are different types of solutions supporting doctor-patient interaction including medical portals, web and mobile applications and telemedicine computer systems all of which are more and more useful and accessible. Such applications not only support acquisition of additional information about diseases and their treatments but they also give a great opportunity to tighten relationship between doctors and patients. Furthermore, such applications usually enables patients selfcontrol by providing tests, questionnaires, as well as different types of diaries including medical history, medication and mood registries, which supports remote patient monitoring and allows collecting patients data without seeing a clinician. Such applications are often rely on dedicated equipment which measures specified body functions such as physiological and biochemical parameters, which are sent and synchronized with dedicated databases. Other telemedicine applications are based on data provided directly by patient. Collecting real-life data it is also a great opportunity for doctors and scientists, because these data are usually embedded in the context of patients daily health habits, mood and emotions.

Considering patient perspectives they benefit from telemedicine mostly by gaining an access to cost-effective and convenient medical services supported real-time technologies [7]. Other benefits include reduction of post-operative complications, improved patient self-management, better medication compliance and easier post-acute discharge monitoring. Furthermore, patient survival often depends on pre-hospital management, which may also include telemedical solutions [8]. In literature it has been also shown that disease diagnosing using telehealth is possible and successful [9], [10], [24].

Many examples of successful implementations of telemedicine services can be found in United States. Internationally, major challenges have yet to be overcame, including wide range of economic, legal, ethical, logistical and cultural issues. There is not only a lack of holistic model for scientific evaluation of telemedicine application efficiency, but also system of interacting network of technical solutions and organizational changes have not been well developed yet [4]. Furthermore, scientific evidence of the effects of home telehealth solutions is still rare (especially in Central Europe, Asia and Africa). However, it is possible to observe some trends of increasing telemedicine and e-Health developments. According to a report from consulting firm InMedica, in 2012 308,000 patients around the world were monitored remotely by providers. Medical help included congestive heart failure, diabetes, hypertension, chronic obstructive pulmonary disease, and mental health conditions.
The number of patients receiving any telecare is expected to reach 1.8 million in just 4 years, mostly throughout primary care physicians, as suggested by InMedica reports [11].

Currently, there are many different medical applications available on the market. Their functionalities and graphical user interface (GUI) quality are highly diversified. Properly adjusted set of functionalities ensures good user experience and determine market success of the application. In order to improve quality and usability of upcoming tools, evaluation of existing solutions may provide valuable insight for further developments. Therefore the objective of this study was to analyze functionalities of selected telemedical applications supporting doctors and patients.

\subsection{Functional analysis method}

Most of medical and health applications dedicated to patients have the form of web portals or web and mobile applications. Each type of application has its own rules and specificity. Moreover, different functionalities serving different purposes can be featured while analyzing such tools. That is why comparison and evaluation of medical applications is challenging and it is important to apply adequate analytical methods.

Several methods for the assessment of application development and functional analysis have been described across literature. A.L. Domingues et al. [15] proposed a method which consisted of the following stages: conceptualization of functionalities, planning and client evaluation. Web development requirements were explored by $\mathrm{Gu}$ et. al. [14] and Lee et. al. [12] presented a framework for measuring key attributes of a Web development. Koch [13] proposed comparison of the phases covered by some Web development methods.

In this study functional analysis was performed by means of top down technique composed of four steps (Figure 1):

1. First, telemedical applications are analysed by means of the ontology of stored and processed data.

2. Based on data gathered during the first step, applications are divided into several groups. The division is made according to the type of data stored in applications including characteristics related to data processing and data analysis.

3. Next, for each group functional aspect of applications are considered.

4. Finally, applications functionalities are analyzed and compared within groups and between groups.

The proposed method allows for comprehensive overview of applications.including various aspects related to the data, information but also features and functionalities. The assessment is made on technology used, target users, and application's purpose._Furthermore, the method considers basic and additional functionalities including paid features. Inter- and intragroup comparison are also enabled by this analytical approach in order to identify functionalities which are common and unique. 


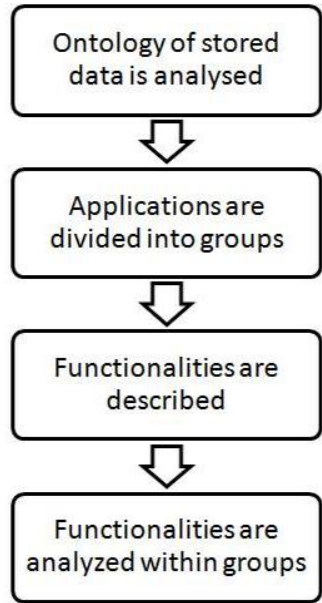

Fig 1. Steps of the functional analysis method

\subsection{Data Ontology Analysis}

The variety of medical and health applications makes it hard to classify and standardize their functionalities. We propose to perform such classification based on type of data stored, character of data flows as well as data ontology.

Proposed ontology was created based of extended analysis of metadata and data flows of eighty different e-health applications supporting patient and/or medical staff. Each application was tested separately in order to collect following metadata:

- users' profiles
patient's data regarding their diseases and moods
. techniques of patient's data obtaining and
presenting
data reporting
. communication techniques
data and experience sharing
medical staff supporting techniques
- educational and information issues.

Obtained metadata were gathered and analysed in order to find common trends and data flows. Based on those results the ontology for e-health applications was proposed. Developed ontology allowed identifying concepts, their attributes and relationships which were used in further steps of the case study. In this study ontology was developed based on the four-step process of Deming cycle [16] (Figure 2):

1. Plan - define objectives and processes necessary to deliver results.

2. Do - implement obtained plan to check its results.

3. Check - study the results and test, if the results are desirable.

4. Act - analyze differences between obtained and planned results and decide, if they are acceptable.

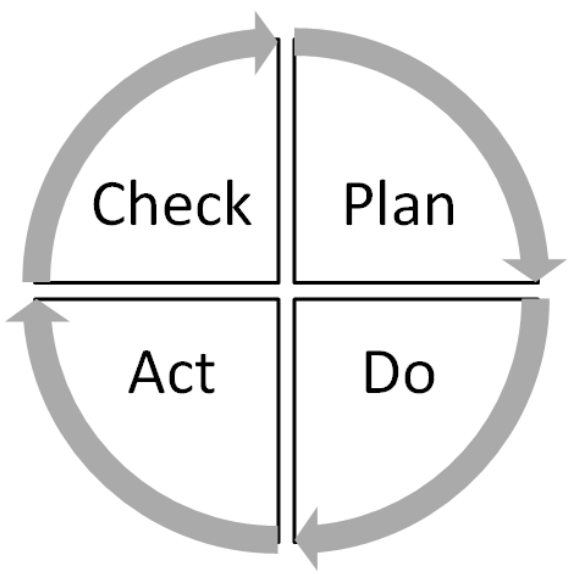

Fig 2. Deming cycle

In more detail, ontology planning consisted of the formation of themes which were based on metadata listed above. The implementation phase was dedicated to identification of relevant terms and based on their relevancy ontology classes were formed.

Top-down technique was applied to distinguish main classes from less relevant ones acting as properties of the main classes. In top-down technique general concepts were included prior to specialized concepts. Relations between terms were also defined. The process of ontology testing was based on a set of questions (called competency questions) [17] focused on e-health applications. Those questions help to check if the ontology covered all relevant information and concepts of e-health applications domain. In the act phase some classes were resituated, some terms were excluded as irrelevant and some relations were redesigned leaving for further analyses the following classes: Patient, Medical Staff, Communication, Self-observation, Treatment, Diagnosis, Educational assets and Medicines base. Based on these classes e-health application ontology was created as shown on Figure 3. Phases were repeated several times and each iteration allowed to precise the model and added more details to it.

According to ontology shown on Figure 3 there are several aspects important for e-health applications analyses. The character of medical and health applications determines their delivery aims: they are dedicated to physicians and other medical staff (for example nurses) as well as patients and their relatives. There are numerous applications collecting data directly from patients who input relevant information based on self-observation records. Such data may include symptoms of the disease, easy to measure parameters (such as pulse rate) or characteristics related to well-being and mood. These data are used in practice in the process of diagnosing, monitoring and reporting outcomes to the patients. Applications used for diagnosis or monitoring between individual medical visits may require gathering more detailed data compared to other applications. There are also application supporting physicians in the process of diagnosis by means of processing delivered information ie. data related to the disease symptomatology and comparing them to entries of dedicated database. Some medical disciplines such as cardiology requires applications equipped with electronic and telecommunications technologies able to register patient's physiological signals and biological parameters such as devices measuring blood pressure or heart rate which transfer the measurement data to application. Medical and health applications are sometimes also complemented by medicines search engines. Some 
applications use patients' data to support medical staff in the process of patients' registration, planning and conducting medical visit. Medical histories of patients also become digitalized with support of dedicated applications and software known as electronic health record. More advanced applications go beyond data sharing and enable online consultations or psychosocial counselling through two-way audio and video technology. This is also a way to ensure the data flow of educational materials dedicated to particular patients or to the specific group of patients. Educational and supportive role of medical applications allows medical staff to provide training and educate patients about diseases, their symptoms and treatment or topics such as human anatomy and physiology. There are also applications that enable communication between patients and physicians or between patients and their relatives as well as amongst patients. These solutions gather larger data and information grouped in various ways usually in dedicated knowledge bases (organized around topics and posts, articles, audio and video content) or in group communication channels (messages, links).

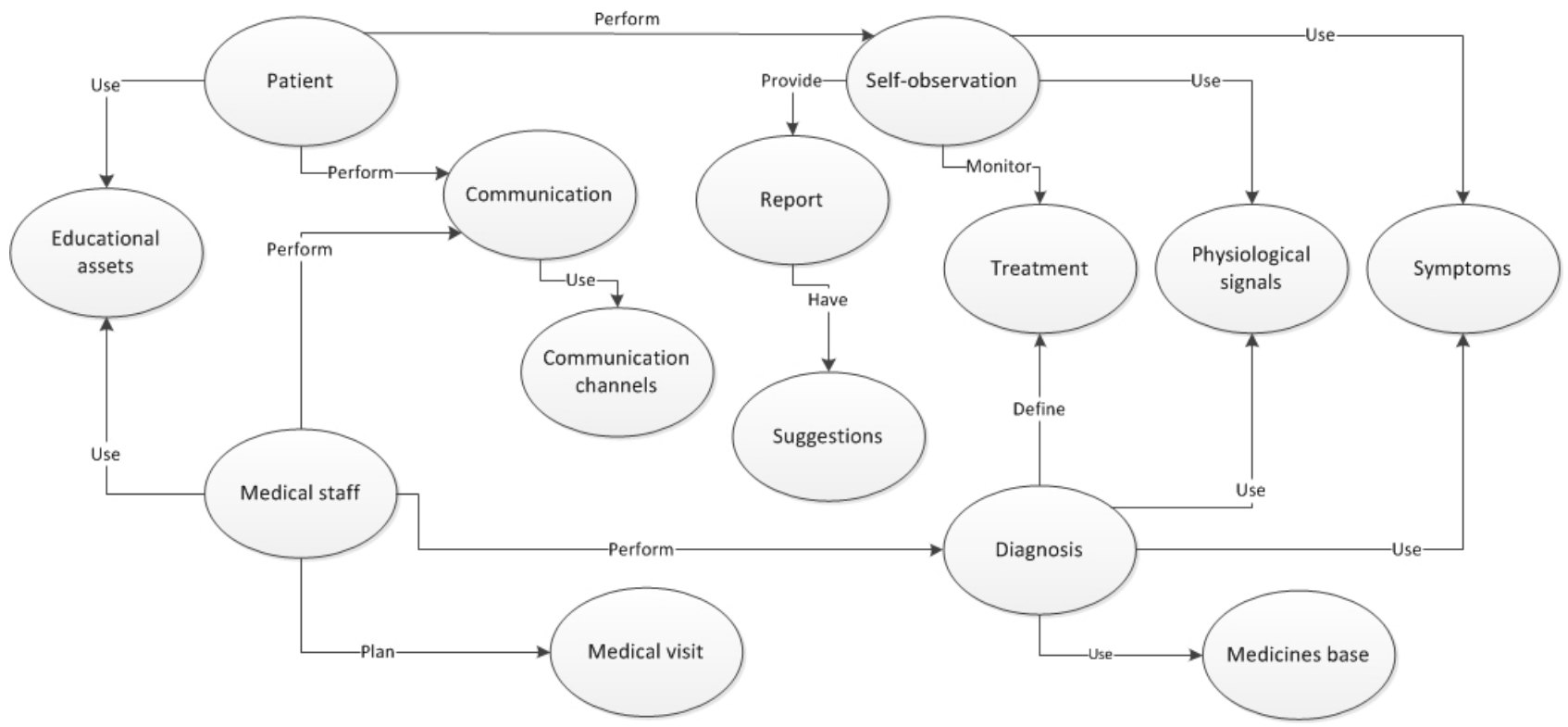

Fig 3. E-health application ontology

Medical applications which are currently available on the market come in vast diversity. Based on analysis of data ontology commonly used in medical applications, here we distinguish three main groups of application:

1. Communication and social networks _-_ - sites gathering interest groups and communities, enabling sharing information.

2. Self-observation, monitoring and diagnosis - tools dedicated to patients, who wants to keep a journal of disease and share it with other people including doctors.

3. Support for medical professionals - applications dedicated to doctors, supporting processes of patient registrations, planning their visit, supporting the process of drug adjustment.

Based on our classification, each of the application included in this study was assigned to the one of mentioned groups and detailed functional analysis was performed.

Presented analysis is a continuation of our former study [25] which aimed to analyze and discuss internet tools supporting commercialization of research results. The paper was focused on functionalities in tools supporting different stages of research results commercialization. Trends and perspectives of business-science cooperation were also presented. The functionality analysis was based on division between the main and additional functionalities. This division was also applied in the presented study. Moreover, there we also analyzed functionalities related to social networking on-line, and we included the same social functionalities in presented study. These fuctionalities were however mainly some general features including user profiles, forum, discussion panels, advanced searching, news and event, data and information sharing, document sharing, message exchange, invitations through social media.

\section{RESULTS OF FUNCTIONAL ANALYSIS}

Diversity of medical applications represents various functionalities which are incorporated in these solutions depending on the purpose for implementing specific tools. Based on ontology analysis we distinguished three major groups of applications (Communication and social networks, Self-observation, monitoring and diagnosis, Support for medical professionals). The next step was to perform functional analysis in order to characterize better each group of medical applications by ascribing to them specific functionalities, which were considered main or additional for specific group. We decided to focus in this study on the web applications. Despite growing popularity of mobile applications, the web applications are those that have wider range of functionalities and are dedicated to wider range of users. However some examples of mobile applications are also mentioned later in our case study analysis. Below we describe the results of functional analysis for each group of medical applications indicating the cases that were used as a material for the analysis.

\subsection{Communication and Social Networks}


There are many medical portals and applications that create social networks of interest groups, mainly patients. These applications are dedicated to patients who want to share with other users their opinions, disease symptoms, experiences and good practices. Basic versions of such applications are forums and discussion groups. However, these applications usually incorporate much more functionalities enabling users to exchange experiences and share problems. Such applications might be dedicated not only to patients but also to other groups of users. There are for instance social network tools that support the relationships between doctors and patients.

Some of these portals are professionalized and provide health services such as e-medical consultations, electronic prescriptions and monitoring the patient's condition.
Mission of E-medical consultations is to give a patient access to medical advice on the topic, without having to leave home. This kind of services usually refers to the situation when patient or other user is interested in obtaining medical advice available on the website. After registration, patient can request e-consultation, usually by selecting a doctor and setting other parameters such as time of consultation. This type of solution very often is based on a model of social validity whereas evaluative feedback regarding service providers is shared between users. E-medical consultations are usually supported with technology which allows delivering scanned documents such as medical history of the patient. Most of E-medical consultations are paid services.

Table 1. Functionalities of communication and social networks applications.

\begin{tabular}{|c|c|c|c|}
\hline No & Functionality name & Main functionality & $\begin{array}{l}\text { Additional } \\
\text { functionality }\end{array}$ \\
\hline 1 & Profiles for all users & $\mathrm{X}$ & \\
\hline 2 & Forum & & $\mathrm{X}$ \\
\hline 3 & Discussion panels & $\mathrm{X}$ & \\
\hline 4 & Advanced searching & & $\mathrm{X}$ \\
\hline 5 & News and events & & $\mathrm{X}$ \\
\hline 6 & Newsletter & & $\mathrm{X}$ \\
\hline 7 & Data and information sharing & $\mathrm{X}$ & \\
\hline 8 & Documents sharing & & $\mathrm{X}$ \\
\hline 9 & Messages exchange & & $X$ \\
\hline 10 & Partner invitations through social media & & $\mathrm{X}$ \\
\hline 11 & Electronic registration for a medical appointment & $\mathrm{X}$ & \\
\hline 12 & Videoconference with a doctor & $\mathrm{X}$ & \\
\hline 13 & Asking a question & & $\mathrm{X}$ \\
\hline 14 & E-history of the disease & $\mathrm{X}$ & \\
\hline 15 & Online medical consultations & $\mathrm{X}$ & \\
\hline 16 & Online prescribing & & $\mathrm{X}$ \\
\hline 17 & Scheduling medical activities & $X$ & \\
\hline 18 & Simple electronic diaries of patients & $X$ & \\
\hline 19 & Sharing electronic diaries of patients & $\mathrm{X}$ & \\
\hline 20 & Electronic reminders & $\mathrm{X}$ & \\
\hline 21 & Share stories & & $\mathrm{X}$ \\
\hline 22 & Locating medical center & & $\mathrm{X}$ \\
\hline
\end{tabular}

Examples of such applications are: Heallow dedicated to the history of a patient's disease and integrated with other medical applications, HemaGo designed to collect and share with doctors and friends patient's data about the history of haemophilia as well as their treatments schedule. Other solutions including tacyjakja.pl, Preventice or Dbees enable patients with chronic disease sharing electronic diaries of symptoms and treatment with their doctors or family members. Applications, such as RealTime Health or Mayo Clinic Patient App, allow sharing stories, experiences and ideas, asking and answering health-related questions, sharing information about local support and programs. There are also 
applications which offer sharing data related to medical activities such as prevention, vaccination or medical examination. Good example of such a tool is mobile application VaxNation which tracks medical history, schedules vaccinations, shares age-appropriate recommendations and helps to locate appropriate clinics and medical centres. All those data might be further shared by linking account to Facebook and Twitter. There are also application dedicated strictly to online medical consultations and prescribing (Myonlinedoctor, Osoz).

\subsection{Self-Observation, Monitoring and Diagnosis}

More and more patients are interested in self-observation, monitoring and diagnosis application. Using such services enables users to perform self- check, monitor treatment progress, keep electronic diary, organize medical visits and follow medication regime.

Such electronic remote control of the patient's condition is usually called E-health service. Such services are usually organized in one of three ways:

- The patient receives an additional electronic device which in case of emergency can send information about specific events to specially established alarm centre. In the centre reported problem is identified and in case of emergency relevant departments are notified (police, ambulance).
- The patient receives more complex electronic device that measures and stores patient health parameters (for example pressure, ECG signal, weight, blood sugar levels) and sends it to the healthcare facility. This allows the doctor to have constant supervision over the patient's health.

- The patient keep his own event log adjusted to the specific disease event log. Data records can be showed to a doctor during a medical appointment. This type of solution enables also to perform selfobservation and to monitor the course of treatment.

Self-observation, monitoring and diagnosis applications have huge prospects for further development. Due to the aging of the population world-wide and rising costs of hospital care the interest in remote health care services will increase.

Remote monitoring of health has a positive impact on patients safety and help to reduce costs of medical care by decreasing length of hospital stay. Patients who are allowed to recover at home with support of medical application may further benefit from reduced stress and mental burden associated with hospitalization. This also apply to carers and families of the patients. E-health is also an interesting option for single people and people with disabilities, who very often depend on social welfare institutions as their care providers.

Table 2. Functionalities of self-observation, monitoring and diagnosis applications.

\begin{tabular}{|c|c|c|c|}
\hline No & Functionality name & Main functionality & Additional functionality \\
\hline 1 & Profiles for all users & $\mathrm{X}$ & \\
\hline 2 & Forum & & $\mathrm{X}$ \\
\hline 5 & News and events & & $\mathrm{X}$ \\
\hline 6 & Newsletter & & $\mathrm{X}$ \\
\hline 7 & Data and information sharing & $\mathrm{X}$ & \\
\hline 10 & Partner invitations through social media & & $\mathrm{X}$ \\
\hline 23 & Medical self-tests and surveys & $\mathrm{X}$ & \\
\hline 24 & Reporting & $\mathrm{X}$ & \\
\hline 14 & E-history of the disease & $\mathrm{X}$ & \\
\hline 15 & Online medical consultations & & $\mathrm{X}$ \\
\hline 17 & Scheduling medical activities & $\mathrm{X}$ & \\
\hline 18 & Electronic diaries of patients & $\mathrm{X}$ & \\
\hline 19 & Sharing electronic diaries of patients & $\mathrm{X}$ & \\
\hline 20 & Electronic reminders & $\mathrm{X}$ & \\
\hline 25 & Medical activities calendars & $\mathrm{X}$ & \\
\hline 21 & Share stories & & $\mathrm{X}$ \\
\hline
\end{tabular}

The most typical examples of self-observation, monitoring and diagnosis applications are: MyAsthma, Viary, The Mindfulness App or AsthmaSense. They offer extended electronic diary supporting patients' activities and motivating them to the fight against disease. Those applications provide also reports and measurement of patients' progress in fighting against disease. There are also applications that implement medical self-tests supporting self-diagnosis of patients who suffer from a particular disease (This Way App, Isabel App). Some application may support more comprehensive overview of the disease whereas other applications focus on selected aspects of particular condition (Depression Mood Monitor). 


\subsection{Support for Medical Professionals}

Applications supporting medical professionals are usually dedicated for doctors and supporting medical staff to help them with following activities:

- Patients registration for a medical visit,

- Keeping medical history of patients,

Planning medical visit of patients,

- Diagnosing process,

- Adjustment of drug types and doses,

- Drugs searching,

- Advanced human body anatomy visualizations.

Supporting applications became more and more popular among medical personnel and medical centres increasingly invest in such systems. There are many benefits of using of such applications including increased work efficiency, improved organization and easier knowledge sharing.

Good examples of such application is ezderm.com. Application supports contact with patient using integrated Phone and videoconferences, complemented by voice recognition function. It also offers scheduling module supporting documentation of patient's visit including symptoms and diagnosis records which can be visualized as 3D anatomical model of the body.

There are also solutions which focus on supporting and integrating telemedicine procedures and applications from different units and levels of medical care. There are portals gathering and sharing medical cases (cases.telemedycyna.wlkp.pl) as well as applications performing early detection of abnormal vital signs based on trend observed using specialized equipment (dynavision.com).

Medical professional can also get support from thematic portals which are usually dedicated to particular medical specialties and are develop to facilitate discussions, knowledge sharing and perform e-consultations (doctormed.pl, kardiolo.pl, ilekarze.pl). Other portals and applications assist in process of diagnosing as well as in process of checking, comparing and adjusting medicines (ehealthme.com, medscape.com, epocrates.com, skyscape.com).

Table 3. Functionalities of applications supporting medical professionals

\begin{tabular}{|c|c|c|c|}
\hline No & Functionality name & Main functionality & Additional functionality \\
\hline 1 & Profiles for all users & & $\mathrm{X}$ \\
\hline 26 & Scheduling module & $\mathrm{X}$ & \\
\hline 2 & Forum for professionals & & $\mathrm{X}$ \\
\hline 3 & Discussion groups & & $\mathrm{X}$ \\
\hline 27 & Disease and condition reference & $\mathrm{X}$ & \\
\hline 28 & Professional articles & & $X$ \\
\hline 29 & Medical education courses & & $\mathrm{X}$ \\
\hline 30 & Drugs lexicon & $X$ & \\
\hline 31 & Drugs adjusting, comparing and searching & $X$ & \\
\hline 32 & Automated diagnosing support & $X$ & \\
\hline 33 & 3D anatomical model of the body & & $\mathrm{X}$ \\
\hline 34 & Patients registration support & $\mathrm{X}$ & \\
\hline 14 & Patients' medical history and symptoms recording & $\mathrm{X}$ & \\
\hline 35 & $\begin{array}{l}\text { Patients' medical history and symptoms processing and } \\
\text { analysing }\end{array}$ & & $\mathrm{X}$ \\
\hline 36 & Voice recognition & & $\mathrm{X}$ \\
\hline 12 & Teleconsultation platform & $X$ & \\
\hline 23 & Assisting in early detection performing & $\mathrm{X}$ & \\
\hline 37 & Recording medical signals using telemedicine equipment & $\mathrm{X}$ & \\
\hline
\end{tabular}

Figure 4 presents the functionalities unique and common for three groups of applications that we distinguished in this study: Communication and social networks, Self-observation, monitoring and diagnosis, and Support for medical professionals.
According to presented results there are several functionalities common to all three groups (Profiles for all users, Forum, Ehistory of the disease), which are directly related to user activity and medical records (profiles, e-history) or play complementary role (forum). 
Communication and social networks shared most functionalities with and self-observation, monitoring and diagnosis applications. These common functionalities include keeping and sharing electronic diaries of patients (with different levels of detail), online medical consultations, scheduling medical activities. Among common feature there were also those which are typical features in most web application namely news and events feeds, newsletters, data and information sharing, partner invitations through social media and electronic reminders. Such results indicate that both groups, communication and social networks, and selfobservation, monitoring and diagnosis applications, although have different purposes and profiles of end users, have number of common secondary features. It is plausible that in future those two groups will become even less distinguishable due to increasing applicability of social network features in web applications but also because of increasing popularity of e-health adds and tools which are becoming increasingly integrated with complementary solutions.
The third group, applications supporting medical professionals, has less common features with remaining groups. We identified only one common feature with selfobservation, monitoring and diagnosis applications (Assisting in early detection performing), which might be helpful for physician during the process of treatment. There are two features common between communication and social networks, and support for medical professionals: Discussion groups and teleconsultations, which play rather complementary role. More pronounced differences of functional characteristics between applications supporting medical professionals and other groups of applications indicate that functional diversification of medical application depends largely on the profiles of end-user and their specific activities that can be supported by the application. Applications dedicated only to medical staff are highly professionalized and focused mainly on supporting specific procedures related to medical and health care services.

\section{Self-observation, monitoring and diagnosis}

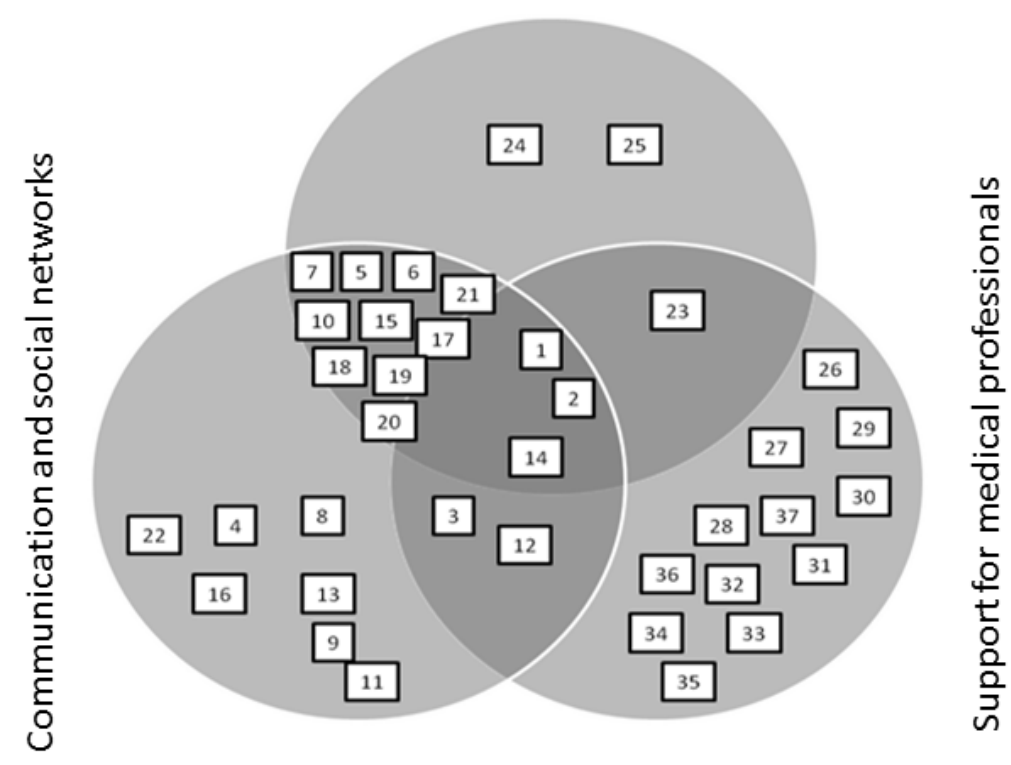

Fig 4. Group comparison of functionalities common and unique for medical applications

\section{SUMMARIZING DISCUSSION}

Presented case study considered functionalities of medical and telemedical applications supporting both, patients and doctors. Our analysis provided overview of most widely- used tools and their functionalities. We discriminated three important groups of telemedical applications which were further characterized by ascribing specific functionalities considering also main and supporting role of these functionalities in each group. Our analysis resulted in extensive yet non-exhaustive list of functionalities.

Tools that were considered in this study incorporated various functionalities that were designed to serve different purposes. There were applications which support communication between patients and doctor including visit planning and treatment monitoring. Some solutions also support diagnosing process, drug searching as well as socialising and sharing experience. Such tools might be used to support patients between their medical visits and can also become a key element of medical care. In fact, sharing problems and finding | support can be sometimes -easier over on Internet then in real life. Internet on one hand can secure anonymity and on the other provides the access to global community of patients and carers seeking and providing support and sharing information about disease. Thanks to knowledge sharing functionalities, Internet has become important platform supporting instant education about diseases and health care. When we consider medical applications available on the market it can be concluded that telemedical solutions have became integrated part of contemporary health care and medical services. These modern tools play important role supporting different processes of health care value chain. According to the findings from our study the major criterion that allows to disentangle the heterogeneity of medical application functionalities is professionalization of the medical application. We found out that the applications which are dedicated to medical professionals and also those application which support patient with professional medical care, incorporate more specialized and specific functionalities. On the other hand there are many functionalities that are less advanced technologically.

Dynamically increasing number of web and mobile medical applications supporting patients and clinicians and grow in popularity of these solutions indicate their important position 
on the market. Medical and health applications give patients overall satisfaction reached in general more than 90\% [18], [19], what makes them useful and worth of investment. Ability to connect with other users provides patients with great opportunities to get knowledge as well as share with other users their problems, opinions, questions and doubts. Patients can also check their health condition and share it with different medical personnel such us physicians, specialists and other health care providers using telecommunications technologies and getting personalized care. However, there are several aspects, which need to be considered. Important one are safety and quality issues. Use of internet technologies requires proper protection of patients' sensitive data to make medical and health applications reliable. In particular it applies to self-observation, monitoring and diagnosis applications, where users report their health condition.

Another important success factor of telemedicine is proven cost saving effect on medical care when compared with traditional approach. Medical and health applications [20] were shown to achieve lower medical and pharmacy costs as well as more efficient service delivery [21]. Some factors that explain economical benefits of implementing telemedical solutions include improved and continuous health monitoring of patients which allows on time intervention minimizing complications of disease. There are several studies which have confirmed improved health care amongst patients using telemedical applications [22], [23]. Furthermore, it has been also shown that such applications enable to obtain clinical outcomes comparable to in-person care [20]. Notwithstanding important role of medical applications in providing a health care these solutions are still considered complementary to traditional ways of providing medical services i.e. through stationary medical centres, clinics and hospitals. However, the role of telemedical care is expected to following technological developments. We would like to point out that there is already some unexplored potential on the medical applications market with limited number of disease prevention tools. Considering many functionalities such as self-observation, monitoring and knowledge sharing, telemedical solutions present themselves relevant for supporting disease prevention. This can be possibly explained by the fact that telemedicine focused first on most recognized and appealing needs which are related to already expressed disease. Economical reasons may also play important role since the applications are usually commercial products and it is still easier to get customers pay for the care rather than prevention. However it is certain that more innovative telemedical solutions are on their way and classifications of applications and their functionalities presented in this paper will need regular revision and update.

\section{CONCLUSIONS AND FUTURE PLANS}

The paper presents results of functional of modern telemedical applications. Presented study shows the diversity of such applications. Furthermore, the growing popularity of telemedicine will certainly result in development of new, more innovative applications supporting doctors, patients and their relatives. Study should be further extended by more in depth analysis of telemedical applications market including trends and needs of end users as well as a developments of enabling technologies. What is more, authors work on development of own telemedical solution dedicated to support patients with depressive disorders.

\section{REFERENCES}

[1] Marcinko, D. E. and Hetico H. R. Dictionary of Health Information Technology and Security. Springer Publishing Company, New York, 2007.

[2] American Telemedicine Association. What is Telemedicine? http://www.americantelemed.org/abouttelemedicine/what-is-telemedicine\#.VCs7CPl_t8E (accessed 1 October 2014)

[3] Bashshur, R. L. Telemedicine and health care, Telemedicine Journal and e-Health, 2002, 8 (1), pp.5-12.

[4] Koch, S. Home telehealth - current state and future trends, International Journal of Medical Informatics, 2006, 75(8), pp. 565-76.

[5] Rockville M.D. Agency for Healthcare Research and Quality. Patient Safety and Quality: An Evidence-Based Handbook for Nurses, In: Hughes RG (eds). Advances in Patient Safety. 2008, 04.

[6] Kang, H., Mahoney. D., Hoenig. H., Hirth, V., Bonato, P., Hajjar, I. and Lipsitz, L. In situ monitoring of health in older adults: technologies and issues. J Am Geriatr Soc, 2010, 58(8), pp.1579-86.

[7] Video Conferencing Advice. Overview of Major Telemedicine Solutions. http://www.videoconferencingadvice.com/telemedicinesolutions.html (accessed 12 October 2014)

[8] Ackerman, M., Craft, R., Ferrante, F., Kratz, M., Mandil, S. and Sapci, H. Chapter 6: telemedicine technology. Telemedicine Journal and e-Health, 2002, 8(1), pp.7178.

[9] Schwaab, B., Katalinic, A., Riedel, J. and Sheikhzadeh, A. Pre-hospital diagnosis of myocardial ischemia by telecardiology: Safety and efficacy of a 12-lead electrocardiogram, recorded and transmitted by the patient. J Telemed Telecare 2005;11(1), pp.41-4.

[10] Belachel, N., Belacel, N., Wang, Q. and Richard, R. Web-integration PROAFTN methodology for acute leukemia diagnosis. Telemed J E Health 2005, 11, pp. 652-9.

[11] Hertz B. T. Telemedicine - patient demand, cost containment drive growth. Medical Economics. http://medicaleconomics.modernmedicine.com/medicaleconomics/news/modernmedicine/modern-medicinefeature-articles/telemedicine-patient-demand-c accessed 20 October 2014)

[12] Lee, H., Lee, C. and Yoo, C. A scenario-based objectoriented methodology for developing hypermedia information systems. Proceedings of the 29th Hawaii International Conference on System Science, 1998, 5, 53-61.

[13] Koch, N. Software Engineering for Adaptive Hypermedia Systems: Reference Model, Modeling Techniques and Development Process. PhD thesis, Ludwig-Maximilians University, Germany, Dec. 2000.

[14] Gu, A., Henderson-Sellers, B. and Lowe, D. Web modelling languages: the gap between requirements and current exemplars. In Proceedings of the 8th Australian World Wide Web Conference, Gold Coast, pp. 362-375, 2002. 
[15] Domingues, A.L., Bianchini, S.L., Costa, M.L., Ferrari, F.C. and Maldonado, J.C. Web application development methods: a comparison. 'Workshop on Business Process Management', 2007.

[16] Deming, W. Edwards. Out of the Crisis. MIT Center for Advanced Engineering Study, 1986.

[17] Dritsas, S., Gymnopoulos, L., Karyda, M., Balopoulos, T., Kokolakis, S., Lambrinoudakis, C. and Katsikas, S. A knowledge-based approach to security requirements for e-health applications. The electronic Journal on Emerging Tools and Applications, In the Special Issue: "Emerging Security Paradigms in the Knowledge Era, 2006, 2 (1)

[18] Gustke, S.S., Balch, DC., West VL and Rogers LO. Patient satisfaction with telemedicine. Telemedicine Journal Spring, 2000, 6(1), pp.5-13.

[19] Brodey, B.B., Claypoole, K.H., Motto, J., Arias, R. and Goss, R. Satisfaction of forensic psychiatry patients with remote telepsychiatric evaluation. Psych Services. 2000, 51(10), pp. 1305-7.

[20] Cryer, L., Shannon, S. B., Van Amsterdam, M. and Leff, B. Costs For 'Hospital At Home' Patients were 19 percent lower, with equal or better outcomes compared to similar inpatients. Health Affairs. 2012, 31(6), pp.1237-1243.

[21] ATA. Examples of research outcomes telemedicine's impact on healthcare cost and quality, http://www.americantelemed.org/docs/defaultsource/policy/examples-of-research-outcomes--telemedicine's-impact-on-healthcare-cost-andquality.pdf, (2013, accessed 20 October 2014)

[22] Fisher, E. B., Boothroyd, R. I., Coufal, M. M., Baumann, L.C., Mbanya, J.C., Rotheram-orus, M. J., Sanguanprasit, B. and Tanasugarn, C. Peer support for self-management of diabetes improved outcomes in international settings. Health Affairs, 2012, 31, pp.1130-139.

[23] Dellifraine, J. L. and Dansky, K. H. Home-based telehealth: a review and meta analysis. J Telemed Telecare. 2008;14(2), p.62-6.

[24] Mukesh, J. and Durgesh, P. Proposed E-Health Service Model by using Smart Phone Apps for Uttarakhand. International Journal of Computer Applications 97(19):14-17, 2014.

[25] Plechawska-Wojcik, M. and Rybka, J. Analysis and comparison of tools supporting commercialization of research results. Actual Problems Of Economics 7(145), 412-423, 2013 